\title{
FACES - A database of facial expressions in young, middle-aged, and older women and men: Development and validation
}

\author{
Natalie C. Ebner, Michaela Riediger, and Ulman Lindenberger \\ Max Planck Institute for Human Development, Berlin, Germany
}

\begin{abstract}
Faces are widely used as stimuli in various research fields. Interest in emotion-related differences and ageassociated changes in the processing of faces is growing. With the aim of systematically varying both expression and age of the face, we created FACES, a database comprising $N=171$ naturalistic faces of young, middle-aged, and older women and men. Each face is represented with two sets of six facial expressions (neutrality, sadness, disgust, fear, anger, and happiness), resulting in 2,052 individual images. A total of $N=154$ young, middleaged, and older women and men rated the faces in terms of facial expression and perceived age. With its large age range of faces displaying different expressions, FACES is well suited for investigating developmental and other research questions on emotion, motivation, and cognition, as well as their interactions. Information on using FACES for research purposes can be found at http://faces.mpib-berlin.mpg.de.
\end{abstract}

Faces with neutral or emotional expressions are frequently used as experimental stimuli in a wide array of research areas, including perception, attention, memory, social reasoning, emotion, infant and adult development, and neuroscience. One reason for this broad interest in faces as research stimuli is that they constitute a unique category of objects. From very early on in life, all sighted individuals have manifold experiences with faces. Some evidence suggests that faces, in contrast to other visual objects, are preferentially processed in specific areas of the brain such as the fusiform gyrus (Gross, Rocha-Miranda, \& Bender, 1972; Haxby et al., 1994; Kanwisher, McDermott, \& Chun, 1997; Puce, Allison, Gore, \& McCarthy, 1995; cf. Tovée, 1998).

A number of studies have shown that variations in such characteristics as the face's expression, age, gender, or race can influence how the face is evaluated, processed, and remembered and that this can vary for perceivers of different ages (Bäckman, 1991; Brigham \& Barkowitz, 1978; Ebner, 2008; Golby, Gabrieli, Chiao, \& Eberhardt, 2001; Isaacowitz, Wadlinger, Goren, \& Wilson, 2006; Mason, 1986; Mather \& Carstensen, 2003; Ruffman, Henry, Livingstone, \& Phillips, 2008). Studies have, for instance, shown that older adults are less able to identify facial expressions than are young adults (for a meta-analysis, see Ruffman et al., 2008), that they have better memory for positive than for negative faces (Mather \& Carstensen, 2003), and that they attend less to negative than to neutral faces (Isaacowitz et al., 2006). These studies have almost exclusively used emotional faces of young individuals and have not systematically varied the age of the face, even though there is evidence that faces that are more like the face of the individual studying them are recognized better than faces that are different. Specifically, evidence for an own-age bias in face recognition and person identification suggests that adults of different ages are more likely to recognize faces of their own age group than faces and persons of other ages (Anastasi \& Rhodes, 2006; Bäckman, 1991; Bartlett \& Fulton, 1991; Lamont, Stewart-Williams, \& Podd, 2005; Wright \& Stroud, 2002). The own-age bias is generally thought to be due to the amount of exposure an individual has to certain classes of faces, in the sense that people typically see faces similar to their own more frequently and might, therefore, be more familiar with them (Bartlett \& Fulton, 1991).

This similarity effect between the face and the perceiver complicates the interpretation of the results of most of the age-comparative studies using facial stimuli that have been conducted up to now. Due to a lack of appropriate stimuli, most studies have not systematically varied the age of the presented faces. Consequently, older adults, for instance, may have been at a disadvantage, relative to young adults, when asked to process and recognize young faces. It is therefore of crucial importance to use faces of different ages when comparing face processing in individuals of different ages.

A first major step in overcoming this lack of appropriate facial stimuli has been undertaken by Minear and Park (2004), who created a new large set of faces representing a wide range of different age groups: the Productive Aging Laboratory (PAL) Face Database. This life span database of adult facial stimuli includes over 1,000 color

N. C. Ebner, natalie.ebner@yale.edu 
(and black-and-white) photographs of adults ranging from 19 to 93 years of age. These faces can be downloaded for research purposes from https://pal.utdallas.edu/facedb/. The database contains neutral facial expressions for each individual face (for norms of a subset of images from this database, see Ebner, 2008, and Kennedy, Hope, \& Raz, 2009). For several of the faces of different ages, there are also happy, sad, angry, annoyed, grumpy or disgusted, and surprised facial expressions. However, so far this database does not comprise a full cross-classification of facial expressions and individual faces, in the sense that each face is represented with each of several different facial expressions. Other existing databases cover various facial expressions for each individual face (see, e.g., Calvo \& Lundqvist, 2008; Palermo \& Coltheart, 2004). Examples are the Pictures of Facial Affect (PoFA; Ekman \& Friesen, 1976), the Karolinska Directed Emotional Faces System (Lundqvist, Flykt, \& Öhman, 1998), the Yale Face Database (n.d.), and the Psychological Image Collection at Stirling (University of Stirling Psychology Department, n.d.). None of these emotional stimuli databases, however, contains images of faces of different age groups - and of older adults, in particular.

The aim of our project was to go beyond existing databases of adult facial stimuli by creating a large set of images of naturalistic faces in which both the age and the expression of the depicted faces are systematically varied, and to validate this new set of pictures. We compiled photographs of young, middle-aged, and older women and men, each displaying six facial expressions: neutrality, sadness, disgust, fear, anger, and happiness. We cooperated with a model agency to recruit participants, a professional photographer to take the pictures in a photo studio at the Max Planck Institute for Human Development in Berlin (MPIB), and a professional digital media designer to postprocess and edit the images. In a subsequent validation study, each face was rated in terms of facial expression and perceived age by young, middle-aged, and older women and men.

\section{METHOD}

\section{Development of the FACES Database}

Face models. The face models were 61 young $(M=$ 24.3 years, $S D=3.5$; age range, $19-31 ; 51 \%$ women), 60 middle-aged ( $M=49.0$ years, $S D=3.9$; age range, $39-55 ; 48 \%$ women $)$, and 58 older $(M=73.2$ years, $S D=$ 2.8 ; age range, $69-80 ; 50 \%$ women) extras, specials, or actors who were, with very few exceptions, recruited through a model agency ("Agentur Wanted," Berlin). To be considered as a face model, a person needed to have an "average type" of look without displaying any prominent, eye-catching features (e.g., beards, tattoos, piercings). Furthermore, he or she had to be able to identify text and pictures presented at a distance of $1-1.5 \mathrm{~m}$ without wearing glasses. All the models were Caucasian.

Prior to the photo-shooting session, the participants were informed via phone, e-mail, or fax that the aim of the project was to set up a database comprising portraits of young, middle-aged, and older adults displaying six different facial expressions for use in scientific research.
Persons who felt capable of expressing each of the six different faces (with the help of face training and with the support of a trained research assistant; see below) were booked for up to $3 \mathrm{~h}$. They were asked not to put on makeup and not to wear clothing covering the neck on the day of the photo-shooting session.

Procedure and Materials. Photo-shooting sessions took place at the MPIB between December 2005 and May 2006 in a studio specifically set up for this purpose. A trained research assistant informed the face models about the general aim of the project and of the day's session, as well as about the specific procedure in the photo studio. The face models were told that they would be photographed various times, showing each of six facial expressions.

The photographer and the photo assistant helped the face models display these facial expressions with a newly developed procedure that comprised three (partly combined) phases: (1) an emotion induction phase, aimed at triggering the spontaneous experience of the respective emotion; (2) a personal experience phase, aimed at inducing the emotion idiosyncratically by imagining personally relevant events that had elicited the respective emotion in the past; and finally, (3) a controlled expression phase, in which the models would receive instructions and go through face training prior to the photo session on how to move and hold specific facial muscles to optimally represent the respective emotion. The face models then signed an informed consent document permitting the use of their pictures for research purposes. At the end of the introduction, gender and age were recorded, and a personal code was assigned to ensure anonymity.

Following that, face models completed the face training. With the help of a manual, based on Ekman and Friesen (2003), the research assistant explained the position of the muscles around the eyes, the nose, and the mouth for an optimal representation of each of the six facial expressions. The manual also contained sample pictures of a young man's face, taken from the PoFA, displaying each of the facial expressions in a prototypical way (neutrality, WF2-5; happiness, WF2-12; sadness, WF3-28; disgust, WF3-11; surprise, WF2-16; fear, WF3-16; anger, WF3-4; Ekman \& Friesen, 1976). Even though evidence suggests that facial expression of an emotion triggers experience of the emotion (Coan, Allen, \& Harmon-Jones, 2001; Duncan \& Laird, 1980; Strack, Martin, \& Stepper, 1988), the face models were told that the aim was not necessarily to actually experience the emotion but, rather, to optimally show it on the face so that other persons could recognize it. They were instructed to display each facial expression as intensively as possible, but in a natural-looking way.

Following these instructions, the face models were asked to take off their jewelry and glasses, to remove makeup and any clothing that covered the neck, and to put on a standard gray shirt. They then had up to $30 \mathrm{~min}$ to practice each of the facial expressions on their own in front of a mirror with the help of the face-training manual and large printouts of the prototypical PoFA face. After about $15 \mathrm{~min}$, the research assistant returned for feedback and further instructions on how to optimize each of the facial expressions. 
After this training phase, the face models entered the photo studio, where they were received by the photographer and the photo assistant. They were then seated in front of a neutral gray (color type, smoke gray) background provided by a portable projection screen on a height-adjustable chair and were instructed to look directly into the teleprompter during photo shooting. The photographer stood behind the teleprompter and the camera. The photo assistant stood next to the photographer, in face contact with the face model. Continuously during the session, both the photographer and the photo assistant gave instructions and feedback. Pictures of the facial expressions displayed by the face models were continuously taken throughout the session.

To warm up, the session started with neutral facial expressions. This was then followed by sad, disgusted, fearful, angry, and, finally, happy faces. To support the models in the optimal display of facial expressions, the earlier described three-step procedure was followed, separately for each expression. The participants were continuously photographed during all three phases (on average, 150-200 pictures were taken per person). In the emotion induction phase, a subset of pictures from the International Affective Picture System (IAPS; Lang, Bradley, \& Cuthbert, 1998) was presented on the teleprompter. These pictures had been preselected on the basis of the criterion to optimally trigger the experience of the respective emotion in support of the facial expression of this emotion. Face models were instructed to just watch the pictures, to experience the emotion it elicited, and to spontaneously show it in the face. The photo assistant controlled the presentation time of the pictures. Pictures were shown for neutrality $(n=5$ pictures; IAPS picture codes: 7002, 7004, 7036, $7175,7205)$, sadness ( $n=5$ pictures; IAPS picture codes: 2095, 2800, 9000, 9040, 9250), disgust ( $n=8$ pictures; IAPS picture codes: $1111,3000,3150,3250,7361,7380$, 9008, 9042), fear ( $n=7$ pictures; IAPS picture codes: $1201,1300,1932,6370,6550,6821,6940,8485)$, and happiness $(n=4$ pictures; IAPS picture codes: 1340 , $1440,1811,8490)$. Since the preselection resulted in no adequate pictures for inducing anger, angry faces were photographed only in Phases 2 and 3, as described next.

After this spontaneous emotion induction phase, the face models were asked to select the IAPS picture that they personally experienced as the best trigger of the respective emotion or as the best aid to facial expression of the respective emotion. This specific picture was then presented as a freeze image while the participants were asked to think of and reexperience a situation in which they personally had felt the emotion and to express it as strongly as possible in their face. Since no IAPS pictures were available to support the experience of anger, the face models were, in this instance, asked to think of and reexperience a situation in their personal past in which they had felt anger and to display it as strongly as possible on their face. In order to facilitate the adequate display of sadness, the face models in the sadness condition additionally watched a movie sequence from The Champ by Franco Zeffirelli (1979) taken from a set of clips developed by Hagemann et al. (1999) to induce different emotions.
Finally, in the third phase, the controlled expression phase, the face models again saw the prototypical PoFA face of the respective emotion on the teleprompter. In addition, the photo assistant read aloud the specific instructions regarding the position of the muscles around the eyes, nose, and mouth for optimally displaying (in a highly controlled way) the emotion as it had been practiced during the face training. The photographer and the photo assistant provided continuous feedback until the desired expression was achieved. Following that, and depending on the quality of the facial expressions already taken and on the amount of time left, the photo shooting continued, combining components of all three phases to individually optimize the display of the respective facial expression.

After the photo-shooting session, the face models were led back to the training room, where they could change again and put back on their jewelry and glasses. They then received financial reimbursement and were thanked for participation in the project. The session typically took about $90 \mathrm{~min}$.

Technical information. High-quality digital photographs were taken with a Sony DSC-F828 camera using a flash $(1,500 \mathrm{WS})$ and a resolution of $2,835 \times 3,543$ pixels. All the pictures were color photographs (color mode sRGB). The lighting conditions were $+20^{\circ}$ frontal lighting diagonally from above, with a $185-\mathrm{cm}$ striplight for soft and smooth illumination, $-20^{\circ}$ brighteners from below, and white balance on neutral gray. The photographs were taken through a teleprompter (custom product for photography; color neutral with special glass, -0.8 aperture stops, 15 -in. picture diagonal). The horizontal lens axis was set on the tip of the nose. Focal distance was $120 \mathrm{~mm}$ on $35 \mathrm{~mm}$. At the end of each session, all the images of a given participant were downloaded to a computer and given file names indicating personal code, age, gender, and the targeted facial expression.

Picture selection procedure. The aim of the picture selection process was to identify and select the two images for each person that best represented each of the six facial expressions. In a first step, the photo assistant conducted a preselection on the basis of photographic aspects, such as image definition. Images were also dropped when the shoulders or the head were overly tilted from the vertical, when the face was not forward-facing, when other body parts such as hands or fingers were in the picture, or when the facial expression deviated strongly from the preestablished guidelines. Trained raters then rated the remaining pictures (around $N=23,000$; approximately $n=130$ pictures per person).

To standardize the rating procedure, the ratings were conducted on the basis of a rating manual. Raters were trained with the help of a rating manual that contained information about the central aim of the rating, as well as specific instructions about the rating procedure. It described the position of the muscles around the eyes, the nose, and the mouth for an optimal representation for each of the targeted facial expressions (cf. Ekman \& Friesen, 2003). In addition, it provided sample faces taken from the PoFA (neutrality, WF2-5, JJ3-4, PE2-4, SW3-3; happiness, WF2-12, JB1-9, JM1-4, PE2-12; sadness, WF3-28, JJ5-5, JM3-11, SW2- 
16; disgust, WF3-11, A1-25, JM2-8, PF1-24; surprise, ${ }^{1}$ WF2-16, JB1-12, JM1-16, SW1-16; fear, WF3-16, JJ5-13, NR1-19, PE3-21; anger, WF3-4, A1-14, NR2-7, MF2-5) displaying the facial expressions in prototypical ways. The manual also contained a note on the occurrence of blended emotions, comprising two or more facial expressions, to make the raters aware of this phenomenon.

Each picture was rated by two raters who had not had any contact with the person in the picture during the photoshooting session. The presentation of faces, one face at a time in a randomized order, was controlled using PsyPoint (Steinkraus \& Ebner, 2006) on Apple Power Mac G5 1.8-GHz computers. For each of the facial expressions (i.e., neutrality, happiness, sadness, disgust, surprise, fear, anger), raters judged whether it was present in the face ( $\mathrm{j}=$ yes, is displayed vs. $\mathrm{n}=$ no, is not displayed). Blending of emotions could be indicated by positively responding to more than one of the facial expressions. If the raters responded that a specific facial expression was depicted in the face, they were also asked to rate its intensity on a 3-point rating scale ("How pronounced is this specific facial expression?"; 1 = not very pronounced, $2=\bmod$ erately pronounced, $3=$ very pronounced $)$.

Following that, we selected images on which both raters agreed in terms of their judgments of (1) purity of facial expression (i.e., both raters agreed on the type of facial expression displayed in the picture and did not rate any other expressions as present in the picture) and (2) high intensity of facial expression (i.e., both raters agreed that the given facial expression was very pronounced). From this reduced set of pictures, the two most prototypical images per person and expression were chosen for the final database in consensus sessions consisting of two to three raters.

Editing and standardization of the pictures. With the aim of optimizing the quality of the pictures and producing greater uniformity within each set of pictures of a person, as well as across all persons, the pictures were postprocessed by the photographer and the photo assistant, as well as by a digital media designer. First, a cutout was selected to standardize the size of the head in the picture, in that equal distances to the image borders were set for the upper head, the ears, and the cuff of the shirt (there were slight deviations due to differences in head sizes, neck lengths, and hairstyle heights). At the same time, the heads were optimally aligned with respect to the image borders to neutralize tilted positions. A fixed gray was set for all the faces to standardize the colors. Final digital picture editing included retouching (of prominent details such as moles, pimples, or gold teeth), color matching, and changing or removing details such as strands of hair in the face. To ensure the same brightness, all the pictures were matched to a predetermined standardized matrix image. Picture editing was done in Adobe Photoshop CS on Apple Macintosh. Finally, the pictures were resized to $819 \times 1,024$ pixel resolution and saved in JPEG format. The original images in JPEG/sRGB format (size, 2,835 $\times$ 3,543 pixels) were archived as well.

The final FACES database. Out of the 179 young, middle-aged, and older face models originally photographed, 58 young $(M=24.2$ years, $S D=3.4$; age range,
Table 1

Face Models Included in the Final FACES Database: Distribution by Age Group and Gender

\begin{tabular}{cccc}
\hline & \multicolumn{3}{c}{ Age Group (Years) } \\
\cline { 2 - 4 } Gender & $19-31$ & $39-55$ & $69-80$ \\
\hline Women & 29 & 27 & 29 \\
Men & 29 & 29 & 28 \\
\hline
\end{tabular}

19-31), 56 middle-aged ( $M=49.0$ years, $S D=3.9$; age range, 39-55), and 57 older ( $M=73.2$ years, $S D=2.8$; age range, 69-80) women and men were selected for inclusion in the final database $(N=171)$. Table 1 presents the total number of persons in the final FACES database, broken down by age group and gender. Eight persons ( 2 young females, 1 young male, 2 middle-aged females, 2 middle-aged males, and 1 older male) were not included in the final database for reasons such as low distinctiveness of facial expressions or prominent features (e.g., bald heads, braces).

Since we selected two images per person and expression, each remaining face model is represented with two sets of six facial expressions (Set A and Set B) in the final FACES database. Pictures of a given facial expression were randomly assigned to one of these two sets. In all, the FACES database thus includes 2,052 individual images. Figure 1 presents sample faces (for additional sample pictures, see http://faces.mpib-berlin.mpg.de).

\section{Validation of the FACES Database}

To validate the FACES database and to provide facespecific information as a reference for researchers selecting face stimuli for specific research purposes, each of the 2,052 pictures of faces in the final FACES database was rated in terms of facial expression and perceived age by young, middle-aged, and older raters.

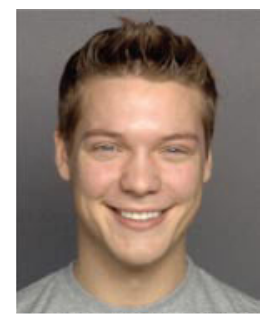

Happy

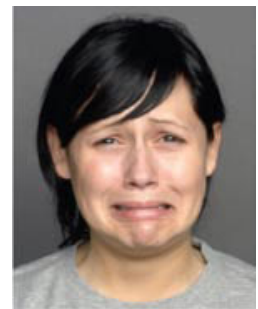

Sad

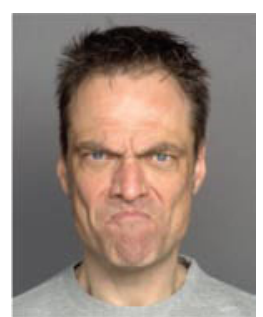

Angry

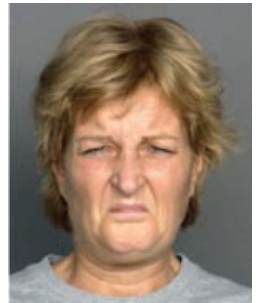

Disgusted

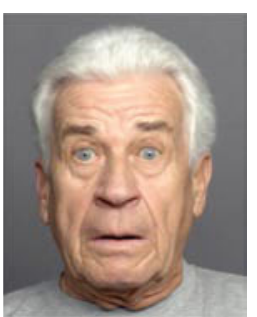

Fearful

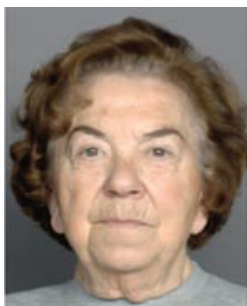

Neutral
Figure 1. Sample faces for the FACES database, which comprises pictures of young, middle-aged, and older women and men, each depicting six different facial expressions. 
Table 2

Description of the Validation Sample

\begin{tabular}{|c|c|c|c|c|c|c|}
\hline & \multicolumn{2}{|c|}{$\begin{array}{l}\text { Young Raters } \\
\quad(n=52)\end{array}$} & \multicolumn{2}{|c|}{$\begin{array}{l}\text { Middle-Aged Raters } \\
\qquad(n=51)\end{array}$} & \multicolumn{2}{|c|}{$\begin{array}{l}\text { Older Raters } \\
\quad(n=51)\end{array}$} \\
\hline & $\begin{array}{l}\text { Women } \\
(n=27)\end{array}$ & $\begin{array}{c}\text { Men } \\
(n=25)\end{array}$ & $\begin{array}{l}\text { Women } \\
(n=25)\end{array}$ & $\begin{array}{c}\text { Men } \\
(n=26)\end{array}$ & $\begin{array}{l}\text { Women } \\
(n=24)\end{array}$ & $\begin{array}{c}\text { Men } \\
(n=27)\end{array}$ \\
\hline \multicolumn{7}{|l|}{ Age (years) } \\
\hline$M$ & 25.7 & 26.2 & 50.5 & 49.4 & 73.7 & 73.5 \\
\hline$S D$ & 2.8 & 3.1 & 3.4 & 3.4 & 3.0 & 2.5 \\
\hline Range & $21-31$ & $20-31$ & $45-55$ & $44-55$ & $70-81$ & $70-78$ \\
\hline Higher secondary level of education ${ }^{\mathrm{a}}(\%)$ & 96 & 84 & 52 & 58 & 46 & 70 \\
\hline
\end{tabular}

aComparable to 2 years or more of college.

Participants in the validation study. A total of $N=$ 154 raters took part in this study. All the participants were Caucasian and native German speakers. The majority of the participants were recruited through the institute's participant pool. Others had heard about the study and had contacted the research group for participation. People who had taken part in related studies involving facial expressions as stimulus material were excluded from participation. Table 2 presents the number and demographic information of the validation sample, broken down by age group and gender. The age by gender groups did not differ in terms of self-reported physical functioning (single item, "How would you describe your current general physical functioning?"; scale of $1-8$, with $8=$ excellent; $M=5.5$, $S D=1.5$ ) but differed in their visual motor processing speed as assessed with the Digit-Symbol Substitution Test (Wechsler, 1981), with young women and men $\left(M_{\mathrm{YW}}=\right.$ $\left.66.3, S D=11.1 ; M_{\mathrm{YM}}=64.0, S D=9.6\right)$ scoring higher than middle-aged women and men $\left(M_{\mathrm{MW}}=46.0, S D=\right.$ 9.1; $\left.M_{\mathrm{MM}}=48.5, S D=14.4\right)$ and older women and men $\left(M_{\mathrm{OW}}=44.8, S D=10.7 ; M_{\mathrm{OM}}=47.7, S D=12.1\right)$ $\left[F(5,143)=18.3, p<.05, \eta_{\mathrm{p}}^{2}=.39 ;\right.$ max score $\left.=93\right]$.

Procedure: Face-rating task. Prior to the start of the study, the participants had agreed to participate in at least three test sessions. Starting with the third session, the participants decided, at the end of each session, whether to continue participation in the study until they had rated all of the 1,026 faces of the set that they had been randomly assigned to (either Set A or Set B). That is, for each participant, the study comprised an individual number of total test sessions $(M=11.28, S D=4.7$; range, $1-24),{ }^{2}$ with older raters $(M=13.53, S D=5.0)$ taking part in more sessions than did young raters $(M=9.4, S D=3.8)$ or middle-aged raters $(M=11.0, S D=4.5)[F(2,148)=11.21, p<.05$, $\left.\eta_{\mathrm{p}}^{2}=.13\right]$. At the beginning of the first session, the participants were informed about the specific testing procedure and signed a consent form. They were then seated in front of computers, separated by partition walls.

The face-rating task started with written instructions and a practice face. The participants were informed that they would be seeing various faces with different facial expressions and that they would have to give their spontaneous, personal judgment for each face in terms of its facial expression ("Which facial expression does this person primarily show?"; response options, in randomized order: neutral, anger, disgust, fear, happiness, sadness) and perceived age ("How old is this person?"; response options: 0-100 years) by moving and clicking the cursor (see Figure 2). Face stimuli were presented, one at a time, in the center at the top of the computer screen. The pictures were approximately $19 \times 16 \mathrm{~cm}$ when displayed on a 19 -in. monitor (screen size, $1,280 \times 1,024$ pixels). Rating dimensions and response options were presented below the image in black on a white background. The faces were presented in randomized order. Each face was shown only once with a certain expression to a participant (i.e., the participants rated either Set A or Set B faces). Stimulus presentation was controlled using custom-made software on Pentium 4 CPU 2.8-GHz computers. After the participants had responded to all rating dimensions for one face, the next face appeared on the screen. To maintain participants' concentration throughout the task, the program stopped for 5-min breaks after every $45 \mathrm{~min}$.

At the end of the first session, the participants responded on the computer to a short sociodemographic questionnaire including one item on physical functioning and worked on the Digit-Symbol Substitution test. In each of the subsequent sessions, the participants continued with the face-rating task. At the end of the study, the participants were debriefed and received a monetary compensation that varied according to the length of their participation in the study (participants who rated an entire set of faces received a monetary bonus in addition).

\section{RESULTS}

\section{Picture-Specific Data}

Each image was rated by a minimum of 8 and a maximum of $14(M=11.0, S D=0.9)$ raters per age group by gender. Two appendixes downloadable from http://faces .mpib-berlin.mpg.de include all of the picture-specific data, with the ratings for each of the faces presented separately. These appendixes detail the participants' categorizations of the facial expression and perceived age of the person in the image. To allow for easy reference, entries are listed according to their identifying labels in the FACES database. First, information referring to the total sample of raters is presented, followed by information pertaining to the age groups by gender of the raters. Specifically, regarding the facial expression ratings, mean percentages of correct expression identification (i.e., mean accuracy) for each of the six expressions separately are reported. Regarding the perceived age ratings, means and standard deviations for perceived age for each of the six expressions 


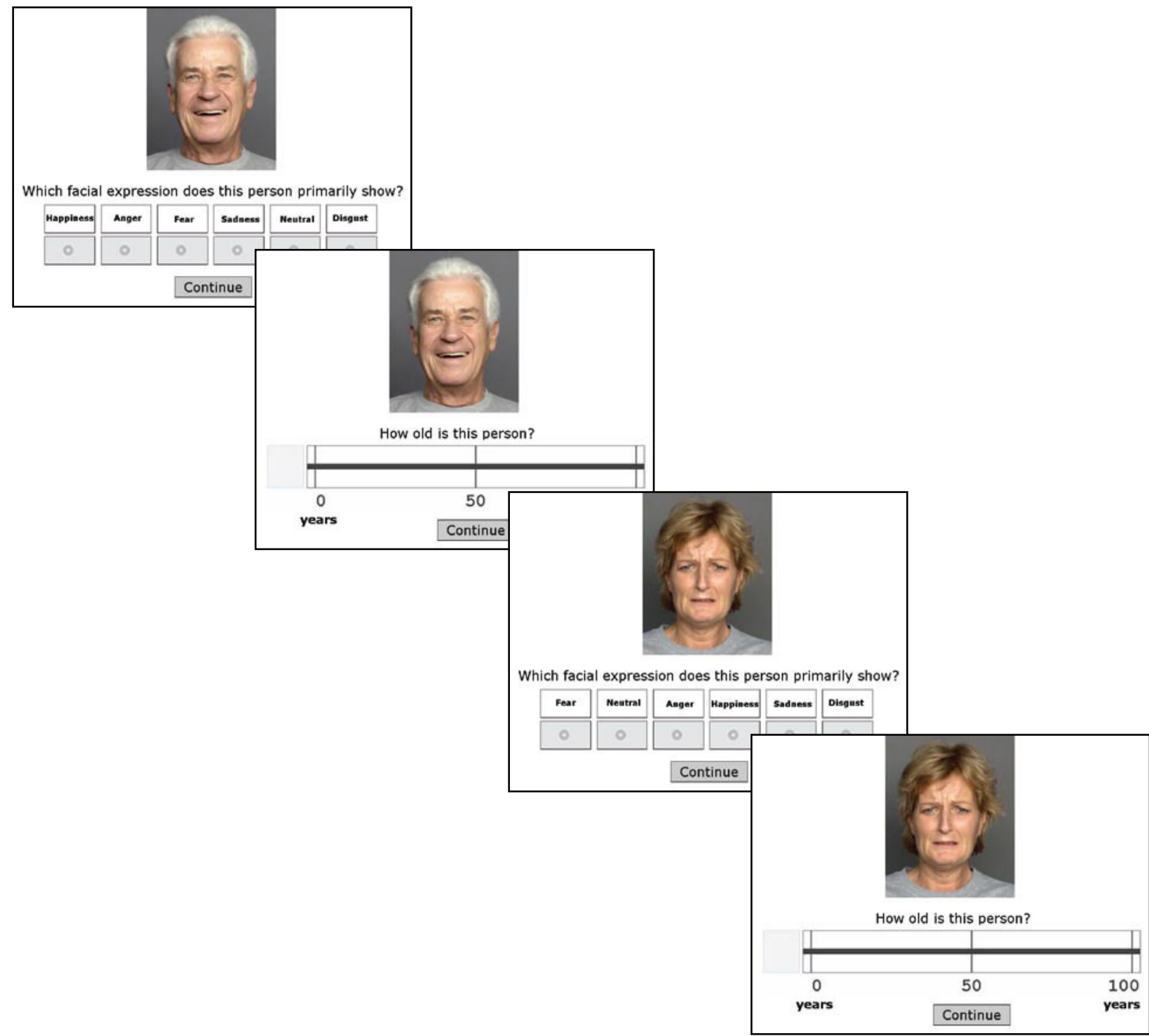

Figure 2. Face-rating task used in the validation study.

separately are reported. This picture-specific information can serve researchers as a basis for selecting pictures that best meet their specific research purposes.

\section{Expression Identification As a Function of}

Age and Expression of Face and Age of Rater

In all the statistical analyses, alpha level was set to .05 . In the total sample of raters, on average, $81 \%(S D=$ $13 \%)$ angry faces, $68 \%(S D=17 \%)$ disgusted faces, $81 \%$ $(S D=15 \%)$ fearful faces, $96 \%(S D=9 \%)$ happy faces, $87 \%(S D=12 \%)$ neutral faces, and $73 \%(S D=14 \%)$ sad faces were correctly identified. As is reflected in the standard deviations, interrater consensus was high. Appendix A presents percentages of correct identification of each of the six facial expressions separately for female and male young, middle-aged, and older faces and for female and male young, middle-aged, and older raters. Overall, percentages of correct facial expression identification in the present study were relatively high and are comparable to those in validation studies of other facial databases (Calvo \& Lundqvist, 2008; Goeleven, De Raedt, Leyman, \& Verschuere, 2008; Palermo \& Coltheart, 2004). This shows that the FACES database comprises a valid set of faces displaying the six different facial expressions.

On the basis of findings that young and older adults differ in their ability to identify facial expressions (Ruffman et al., 2008) and that the age of the face seems to play a role in facial expression identification, we conducted a 3 (age of rater: young, middle-aged, older) $\times 3$ (age of face: young, middle-aged, older) $\times 6$ (facial expression: happy, angry, fearful, sad, disgusted, neutral) repeated measures ANOVA. Figures 3 and 4 present the results. The effects for age of rater $\left[F(2,142)=8.90, p<.001, \eta_{\mathrm{p}}^{2}=.11\right]$, age of face [Wilks's $\lambda=.20 ; F(2,141)=282.73, p<.05, \eta_{\mathrm{p}}^{2}=$ 


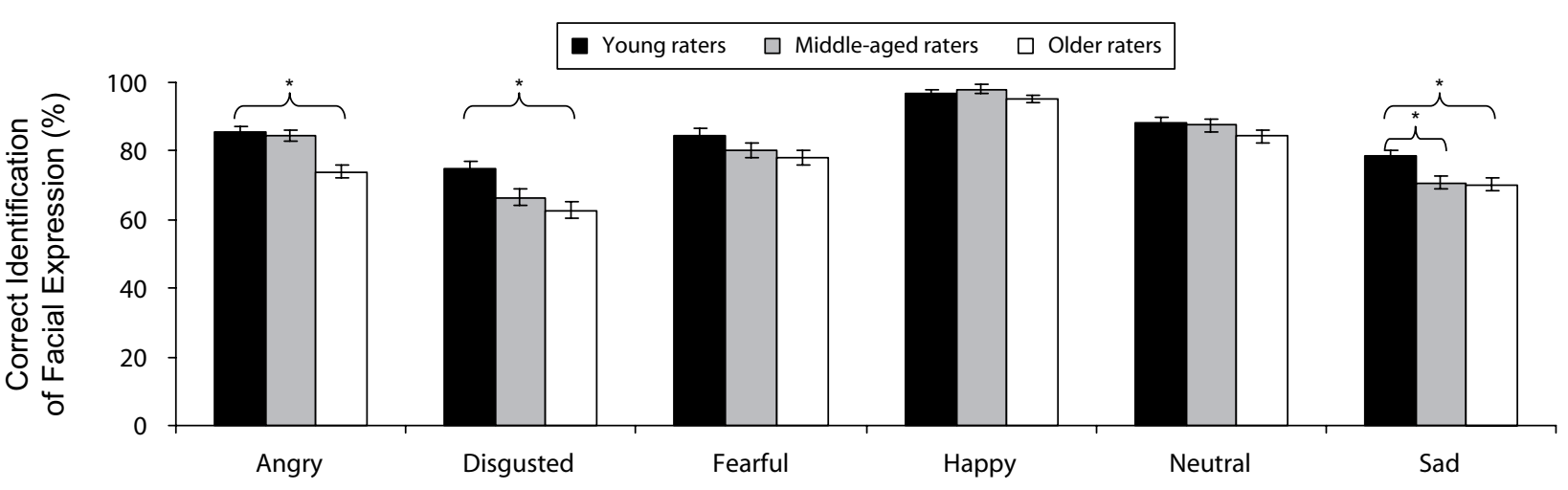

Figure 3. Mean percentages of correct identification of each of the six facial expressions as a function of the age of the raters. Error bars represent standard errors of age-group mean differences. ${ }^{*} p<.05$.

.80 ], and facial expression [Wilks's $\lambda=.16 ; F(5,138)=$ $\left.146.51, p<.05, \eta_{\mathrm{p}}^{2}=.84\right]$, the facial expression $\times$ age of rater interaction [Wilks's $\lambda=.77 ; F(10,276)=3.88$, $\left.p<.05, \eta_{\mathrm{p}}^{2}=.12\right]$, and the age of face $\times$ facial expression interaction [Wilks's $\lambda=.29 ; F(10,133)=32.09, p<.05$, $\left.\eta_{\mathrm{p}}^{2}=.71\right]$ were significant. All other effects did not reach significance $(p>.05)$. To follow up on the significant facial expression $\times$ age of rater interaction, we conducted a 3 (age of rater) $\times 6$ (facial expression) MANOVA collapsed across the different ages of the faces. As is shown in Figure 3, the age groups of the raters did not differ in their ability to identify fearful, happy, or neutral faces. They did, however, differ in their ability to correctly identify angry $\left[F(2,142)=13.62, p<.05, \eta_{\mathrm{p}}^{2}=.16\right]$, disgusted $\left[F(2,142)=6.84, p<.05, \eta_{\mathrm{p}}^{2}=.16\right]$, and $\operatorname{sad}[F(2,142)=$ $\left.5.81, p<.05, \eta_{\mathrm{p}}^{2}=.08\right]$ faces. In line with the literature (e.g., Ruffman et al., 2008), young raters performed more accurately than older raters in identifying anger, disgust, and sadness in faces. They also performed more accurately than middle-aged raters in decoding sad faces.

For each of the expressions, $t$ test comparisons between young, middle-aged, and older faces, collapsed across the different age groups of the raters, followed up on the significant age of face $\times$ facial expression interaction. As is presented in Figure 4, the results showed that, with the exception of fearful faces, expression identification was more difficult for older than for young or middle-aged faces. Furthermore, disgusted, neutral, and sad middle-aged faces were less accurately identified than young faces displaying those expressions. Also consistent with earlier studies (Kirouac \& Dore, 1983; Ruffman et al., 2008), contrast analyses collapsed across young, middle-aged, and older raters showed that identification of happiness was easier than identification of all the other expressions and that identification of disgust was more difficult than identification of all the other expressions in young, middle-aged, and older faces (all $p \mathrm{~s}<.05$; see Figure 4).

\section{Perceived Age As a Function of Age and \\ Expression of Face and Age of Rater}

In the total sample of raters, on average, young faces were rated as $M=28.5$ years of age $(S D=3.5$; age range, $22-42)$, middle-aged faces as $M=49.2$ years $(S D=3.3$; age range, $41-62$ ), and older faces as $M=68.6$ years $(S D=4.1$; age range, $53-80)$, with the actual average ages of young face models being $M=24.2$ years $(S D=$ 3.4; age range, 19-31), of middle-aged face models, $M=$ 49.0 years $(S D=3.9$; age range, $39-55)$, and of older face models, $M=73.2$ years $(S D=2.8$; age range, $69-80)$.

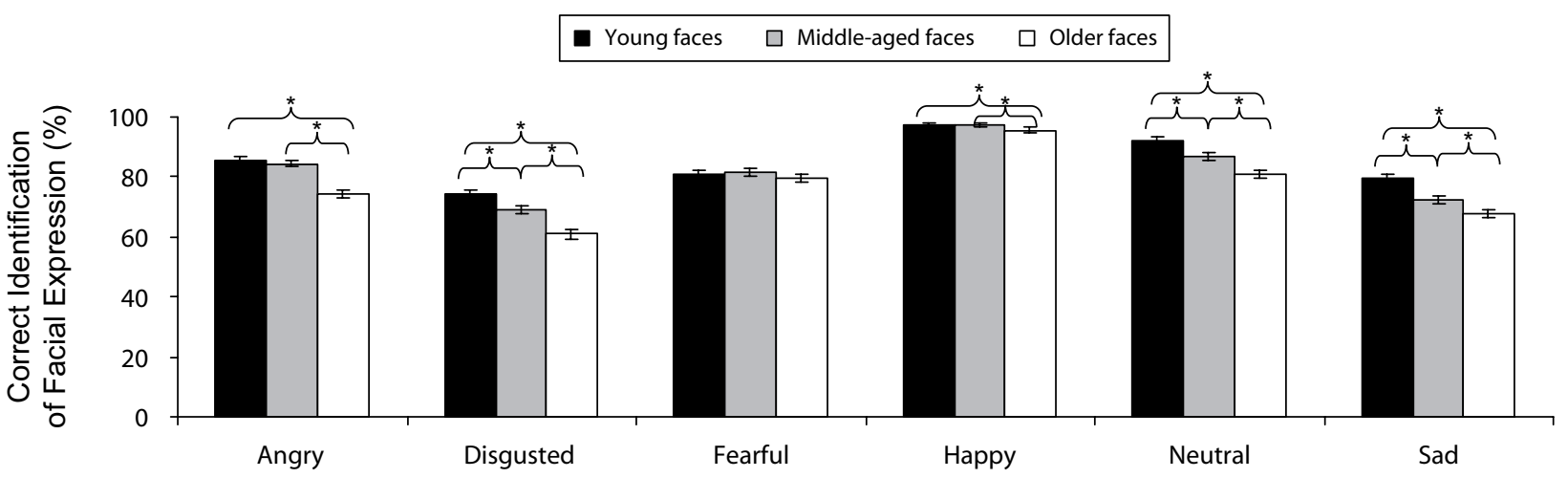

Figure 4. Mean percentages of correct identification of each of the six facial expressions as a function of the age of the faces. Error bars represent standard errors of mean differences between facial expression within young, middle-aged, and older faces, respectively. ${ }^{*} p<.05$. 
The intraclass correlation between the raters was .88, indicating good interrater reliability. Appendix B presents the means of the perceived ages separately for each expression of female and male faces of the different ages and for the age by gender groups of the raters. Even though young and older faces, but not middle-aged faces, were rated slightly older than the actual average ages of young and older face models, these results show that the faces from the FACES database adequately represent the target age groups and, therefore, constitute a valid set of faces in terms of the age of the faces.

With the aim of exploring whether young, middle-aged, and older raters differed in their age ratings of faces of different ages and to examine the influence of different

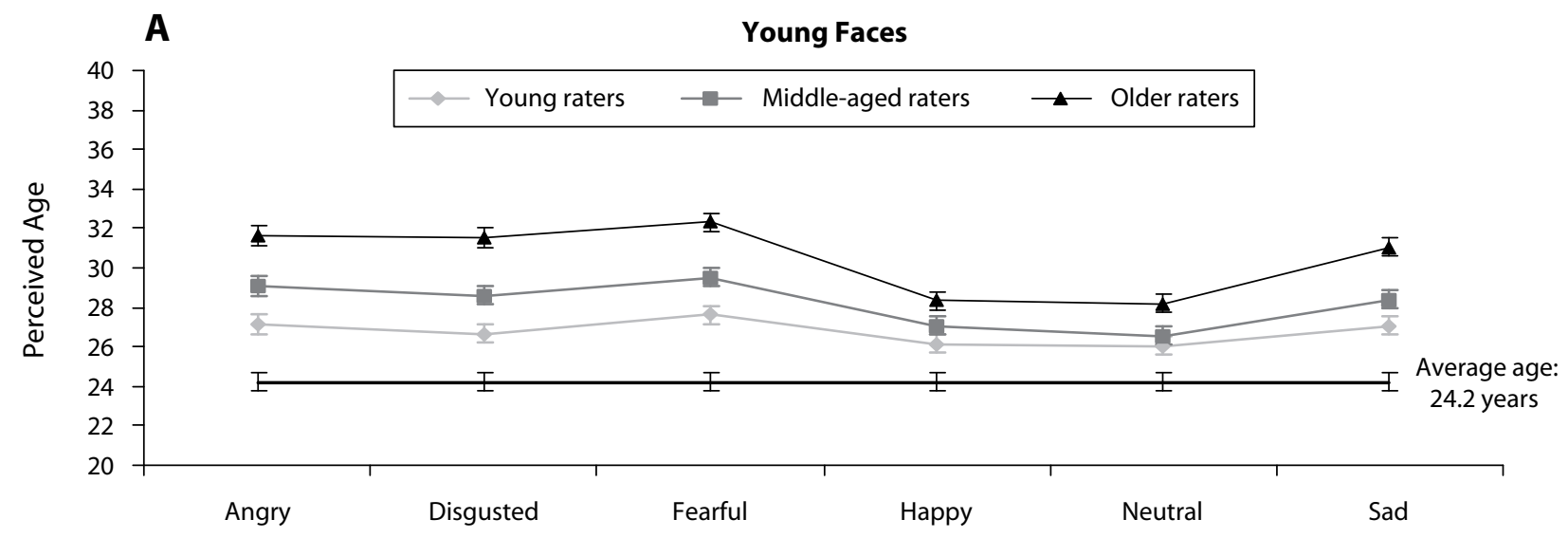

B

Middle-Aged Faces

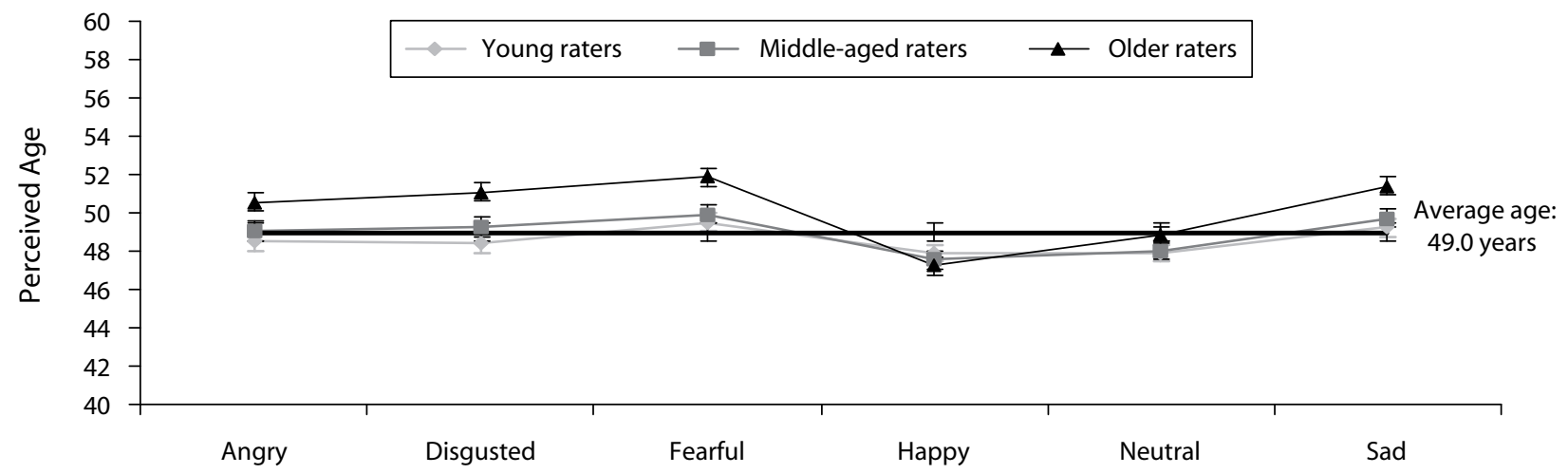

C

Older Faces

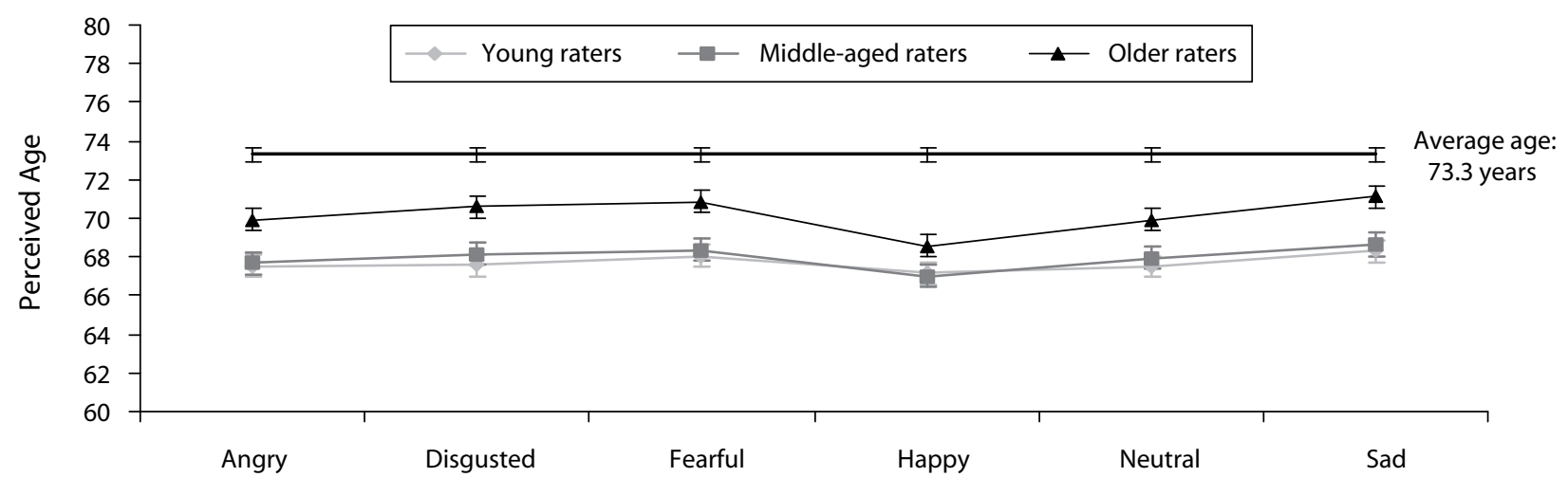

Figure 5. Age of faces as perceived by the participants in the validation study. Error bars represent standard errors of mean differences between age groups and raters. Black straight line indicates average age of (A) young, (B) middle-aged, and (C) older face models. Error bars represent standard errors of mean differences between age groups of raters. Black straight lines indicate the average age of the (A) young, (B) middle-aged, and (C) older face models. 
facial expressions on perceived age, we conducted a 3 (age of rater) $\times 3$ (age of face) $\times 6$ (facial expression) repeated measures ANOVA. The results are summarized in Figure 5. The main effects for age of rater $[F(2,148)=$ $13.11, p<.05, \eta_{\mathrm{p}}^{2}=.15$ ], age of face [Wilks's $\lambda=.02$; $\left.F(2,147)=4,678.80, p<.05, \eta_{\mathrm{p}}^{2}=.99\right]$, and facial expression [Wilks's $\lambda=.16 ; F(5,144)=157.10, p<.05$, $\left.\eta_{\mathrm{p}}^{2}=.85\right]$, as well as the age of face $\times$ age of rater interaction [Wilks's $\left.\lambda=.91 ; F(4,294)=3.62, p<.05, \eta_{\mathrm{p}}^{2}=.05\right]$, the facial expression $\times$ age of rater interaction [Wilks's $\left.\lambda=.49 ; F(10,288)=12.35, p<.05, \eta_{\mathrm{p}}^{2}=.30\right]$, the age of face $\times$ facial expression interaction [Wilks's $\lambda=.31$; $\left.F(10,139)=31.17, p<.05, \eta_{\mathrm{p}}^{2}=.69\right]$, and the three-way interaction between age of face, facial expression, and age of rater [Wilks's $\lambda=.62 ; F(20,278)=3.70, p<.05, \eta_{\mathrm{p}}^{2}=$ .21] were significant.

We followed up on the significant three-way interaction in two ways. First, contrast analyses conducted separately in young, middle-aged, and older faces collapsed across the different facial expressions showed that older raters perceived young faces $\left[M_{\mathrm{OY}}=30.5\right.$ years, $S D=$ 4.2; $\left.F(2,151)=16.76, p<.05, \eta_{\mathrm{p}}^{2}=.18\right]$ and older faces $\left[M_{\mathrm{OO}}=70.2\right.$ years, $S D=4.3 ; F(2,148)=5.96, p<.05$, $\left.\eta_{\mathrm{p}}^{2}=.08\right]$ as older than did young raters $\left(M_{\mathrm{YY}}=26.8\right.$ years, $S D=1.9 ; M_{\mathrm{YO}}=67.7$ years, $\left.S D=3.8\right)$ and middle-aged raters $\left(M_{\mathrm{MY}}=28.3\right.$ years, $S D=3.2 ; M_{\mathrm{MO}}=68.0$ years, $S D=3.8)$. Older raters were less correct in their perceptions of the age of young (i.e., other-age) faces and were more correct in their ratings of the age of older (i.e., own-age) faces than were young or middle-aged raters. There was no effect for middle-aged faces $\left(M_{\mathrm{YM}}=48.6\right.$ years, $S D=2.9$; $M_{\mathrm{MM}}=49.0$ years, $S D=3.4 ; M_{\mathrm{OM}}=50.1$ years, $S D=$ 3.5). All three age groups of raters were good at estimating the correct age of middle-aged faces.

Second, collapsed across the different age groups of the raters and ages of faces, faces with happy expressions $(M=$ 47.4 years, $S D=2.7)$ were rated as younger than faces with other expressions ( $M$ s varied between 47.9 and 49.8 years, $S D$ s varied between 2.7 and 3.1), whereas faces with fearful expressions $(M=49.8$ years, $S D=3.1)$ were rated as older than faces with other expressions $(M \mathrm{~s}$ varied between 47.4 and 49.4 years, $S D$ s varied between 2.7 and 3.1; all $p$ s $<$ $.05)$. With few exceptions, ${ }^{3}$ these effects also held when young, middle-aged, and older raters and young, middleaged, and older faces were investigated separately.

\section{DISCUSSION}

To summarize, we developed and validated a new database of experimental stimuli-the FACES database of facial expressions - that comprises high-quality color photographs of a large number of young, middle-aged, and older female and male naturalistic faces, each displaying six different facial expressions: neutrality, sadness, disgust, fear, anger, and happiness. The FACES database goes beyond existing databases of adult facial stimuli by providing a validated set of images that systematically varies both the age and the expression of the depicted faces, thus encompassing a full cross-classification of facial expressions and individual faces of different ages.
The validation study showed that the percentages of correct facial expression identification for each of the six facial expressions depicted in the images from the FACES database were relatively high and were comparable to those from other facial databases (Calvo \& Lundqvist, 2008; Goeleven et al., 2008; Palermo \& Coltheart, 2004). Furthermore, the perceived age ratings for faces of the three different age groups were adequate and reflected the target age groups. These findings indicate that the newly developed FACES database contains a valid set of faces in terms of facial expressions displayed and age groups represented in the pictures. Furthermore, the present finding that the age of the face, the expression of the face, and the age of the perceiver (and their interactions) have an effect on how well a facial expression is identified and on perceptions of the age of a face, as well as the face-specific information reported in the present study, will be a valuable resource for researchers when selecting faces that are most appropriate for their specific research endeavors.

The FACES database is freely available for use in scientific research. This is an eSciDoc service of the Max Planck Digital Library (MPDL; www.escidoc.org/JSPWiki/en/ Faces). Functionalities of this online service include, among other things, browsing, searching, viewing, selecting, and exporting of FACES pictures. Subsets of selected pictures can be saved for future reference and can also be shared among researchers. Images can be downloaded in the resized resolution of $819 \times 1,024$ pixels in JPEG format or in their original size $(2,835 \times 3,543$ pixels; JPEG format). To prevent circulation of the FACES pictures unrelated to research usage, scientists need to register in order to obtain access to the FACES database. Information on the final FACES database and on registration formalities can be obtained from http://faces.mpib-berlin.mpg.de.

In order to further broaden the applicability of the FACES database as a research tool, an ongoing project led by Sabine Herpertz and Alexander Lischke from Rostock University currently extends the FACES database by modifying a subset of the original images with respect to viewing directions. Specifically, these modifications comprise systematic variation of frontal, right-oriented, and left-oriented direction of gaze. Once these modified faces are finalized, they will be integrated into the eSciDoc service of the MPDL.

Note that all the faces comprised in the FACES database are Caucasian. This limits the applicability of the stimuli. One reason for selecting this homogeneous set in terms of ethnicity of the face was for practicality, since the face models were recruited from a population in Berlin, Germany, in which the majority of inhabitants are Caucasian. Another reason was that our aim was to ensure sufficiently large numbers of faces per category while reducing design complexity. As a consequence, to minimize potential effects of differences in ratings of exclusively Caucasian faces as a function of the origin of the perceiver, we made a special effort to recruit only Caucasian raters for the validation study. In addition, low-level and higher level perceptual features inherent in color naturalistic faces (e.g., hair, wrinkles, complexion), as compared with 
black-and-white schematic faces, need to be considered, depending on the specific research purpose. Furthermore, we report information about ratings of facial expression and perceived age of the faces, but it remains for future work to describe the faces in terms of, for instance, their attractiveness, distinctiveness, and age group differences in ratings with respect to these.

In creating and validating this new extensive set of adult faces and in making it freely available to the research community, we hope to overcome the lack of appropriate stimuli in age-comparative research that limited examination of hypotheses about age of face, facial expression, and their interactions. The FACES database makes possible systematic variation of the age, in addition to the expression, of the presented faces and will allow researchers to address questions on age group differences in, for example, the processing of, attention to, and memory for emotional faces of different ages. Thus, we hope, it will make significant contributions to research in life span development and aging on emotion, motivation, and cognition.

\section{AUTHOR NOTE}

This research was funded by and conducted at the Max Planck Institute for Human Development, Berlin, Germany. The authors thank the photographer, Tom Kretschmer, and the photo assistant, Anja Böhnke, for taking the photographs and preselecting and postprocessing the pictures; Dulce Erdt, Ulrike Altmann, Judith Peth, Susanne Michalke, Paul Muhle-Karbe, Kristin Ackermann, Martina Zellin, and Sebastian Gluth for their assistance in training and supervising the face models and for rating the pictures; the digital media designer, Anka Kammler, for editing the pictures; and the computer programmers, Matthias Bindernagel and Elmar Tampe, for designing and programming an initial version of the FACES software. We are grateful to the eSciDoc project of the Max Planck Digital Library (www.escidoc.org/JSPWiki/en/FACES) for developing, providing, and maintaining the online service that makes the FACES database freely available for scientific research and to Ursula Flitner for coordinating this cooperation. The authors also thank Katja Werheid and Håkan Fischer for discussions about the setup of the database. We also appreciate the time and energy that the face models invested in this project. Correspondence concerning this article should be addressed to N. C. Ebner, Department of Psychology, Yale University, P.O. Box 208205, New Haven, CT 06520-8205 (e-mail: natalie. ebner@yale.edu), or to M. Riediger, Independent Junior Research Group "Affect Across the Lifespan," or U. Lindenberger, Center for Lifespan Psychology, Max Planck Institute for Human Development, Lentzeallee 94, 14195 Berlin, Germany (e-mail: riediger@mpib-berlin.mpg.de or seklindenberger@mpib-berlin.mpg.de).

\section{REFERENCES}

Anastasi, J. S., \& Rhodes, M. G. (2006). Evidence for an own-age bias in face recognition. North American Journal of Psychology, $\mathbf{8}$, 237-252.

BäCKMAN, L. (1991). Recognition memory across the adult life span: The role of prior knowledge. Memory \& Cognition, 19, 63-71.

Bartlett, J. C., \& Fulton, A. (1991). Familiarity and recognition of faces in old age. Memory \& Cognition, 19, 229-238.

Brigham, J. C., \& BARKowitz, P. (1978). Do "they all look alike"? The effect of race, sex, experience, and attitudes on the ability to recognize faces. Journal of Applied Social Psychology, 8, 306-318.

Calvo, M. G., \& LundQvist, D. (2008). Facial expressions of emotion (KDEF): Identification under different display-duration conditions. Behavior Research Methods, 40, 109-115.

Coan, J. A., Allen, J. J. B., \& Harmon-Jones, E. (2001). Voluntary facial expression and hemispheric asymmetry over the frontal cortex. Psychophysiology, 38, 912-925.
Duncan, J. W., \& Laird, J. D. (1980). Positive and reverse placebo effects as a function of differences in cues used in self-perception. Journal of Personality \& Social Psychology, 39, 1024-1036.

Ebner, N. C. (2008). Age of face matters: Age-group differences in ratings for young and old faces. Behavior Research Methods, 40, 130-136.

Ekman, P., \& Friesen, W. V. (1976). Pictures of facial affect. Palo Alto, CA: Consulting Psychologists Press.

EKMAN, P., \& FRIESEN, W. V. (2003). Unmasking the face: A guide to recognizing emotion from facial clues. Cambridge, MA: Malor Books.

Goeleven, E., De Raedt, R., Leyman, L., \& Verschuere, B. (2008). The Karolinska Directed Emotional Faces: A validation study. Cognition \& Emotion, 22, 1094-1118.

Golby, A. J., Gabrieli, J. D. E., Chiao, J. Y., \& Eberhardt, J. L. (2001). Differential responses in the fusiform region to same-race and other-race faces. Nature Neuroscience, 4, 845-850.

Gross, C. G., Rocha-Miranda, C. E., \& Bender, D. B. (1972). Visual properties of neurons in inferotemporal cortex of the macaque. In S. M. Kosslyn \& R. A. Andersen (Ed.), Frontiers in cognitive neuroscience (pp. 83-98). Cambridge, MA: MIT Press.

Hagemann, D., Naumann, E., Maier, S., Becker, G., Lurken, A., \& BARTUSSEK, D. (1999). The assessment of affective reactivity using films: Validity, reliability and sex differences. Personality \& Individual Differences, 26, 627-639.

Haxby, J. V., Horwitz, B., Ungerleider, L. G., Maisog, J. M., Pietrini, P., \& Grady, C. L. (1994). The functional organization of human extrastriate cortex: A PET-rCBF study of selective attention to faces and locations. Journal of Neuroscience, 14, 6336-6353.

IsaAcowitz, D. M., Wadlinger, H. A., Goren, D., \& Wilson, H. R. (2006). Is there an age-related positivity effect in visual attention? A comparison of two methodologies. Emotion, 6, 511-516.

Kanwisher, N., McDermott, J., \& ChUn, M. M. (1997). The fusiform face area: A module in human extrastriate cortex specialized for face perception. Journal of Neuroscience, 17, 4302-4311.

Kennedy, K. M., Hope, K., \& Raz, N. (2009). Life span adult faces: Norms for age, familiarity, memorability, mood, and picture quality Experimental Aging Research, 35, 1-8.

Kirouac, G., \& Dore, F. Y. (1983). Accuracy and latency of judgement of facial expressions of emotions. Perceptual \& Motor Skills, 57, 683686.

Lamont, A. C., Stewart-Williams, S., \& Podd, J. (2005). Face recognition and aging: Effects of target age and memory load. Memory \& Cognition, 33, 1017-1024

LANG, P. J., Bradley, M. M., \& Cuthbert, B. N. (1998). International affective pictures system (IAPS): Digitized photographs, instruction manual and affective ratings (Tech. Rep. A-6). Gainesville: University of Florida.

Lundevist, D., Flykt, A., \& ÖHman, A. (1998). The Karolinska Directed Emotional Faces-KDEF [CD-ROM]. Stockholm: Karolinska Institute Department of Clinical Neuroscience, Psychology Section. Retrieved June 4, 2009, from www.facialstimuli.com/index_files/ Page369.htm.

Mason, S. E. (1986). Age and gender as factors in facial recognition and identification. Experimental Aging Research, 12, 151-154.

Mather, M., \& CARSTEnSEN, L. L. (2003). Aging and attentional biases for emotional faces. Psychological Science, 14, 409-415.

Minear, M., \& Park, D. C. (2004). A lifespan database of adult facial stimuli. Behavior Research Methods, Instruments, \& Computers, 36, 630-633.

Palermo, R., \& Coltheart, M. (2004). Photographs of facial expression: Accuracy, response times, and ratings of intensity. Behavior Research Methods, Instruments, \& Computers, 36, 634-638.

PaL Face Database (n.d.). Retrieved June 4, 2009, from https://pal utdallas.edu/facedb/.

Puce, A., Allison, T., Gore, J. C., \& McCarthy, G. (1995). Facesensitive regions in human extrastriate cortex studied by functional MRI. Journal of Neurophysiology, 74, 1192-1199.

Ruffman, T., Henry, J. D., Livingstone, V., \& Phillips, L. H. (2008). A meta-analytic review of emotion recognition and aging: Implications for neuropsychological models of aging. Neuroscience \& Biobehavioral Reviews, 32, 863-881.

Steinkraus, J., \& Ebner, N. C. (2006). A manual to Psy-Point [Unpublished manual]. Berlin: Max Planck Institute for Human Development. 
Strack, F., Martin, L. L., \& Stepper, S. (1988). Inhibiting and facilitating conditions of the human smile: A nonobtrusive test of the facial feedback hypothesis. Journal of Personality \& Social Psychology, 54, 768-777.

TovéE, M. J. (1998). Is face processing special? Neuron, 21, 12391242

University of Stirling Psychology Department (n.d.). The Psychological Image Collection at Stirling (PICS). Retrieved June 4, 2009, from http://pics.psych.stir.ac.uk/.

WeCHSLER, D. (1981). Manual for the Wechsler Adult Intelligence Scale-Revised (WAIS-R). New York: Psychological Corporation.

Wright, D. B., \& Stroud, J. N. (2002). Age differences in lineup identification accuracy: People are better with their own age. Law \& Human Behavior, 26, 641-654

Yale Face Database (n.d.). Retrieved June 4, 2009, from http://cvc .yale.edu/projects/yalefaces/yalefaces.html.

ZEFFIRELLI, F. (DIRECTOR) (1979). The champ [Motion picture]. United States: Metro-Goldwyn-Mayer (MGM).

\section{NOTES}

1. Since fear and surprise are often blended in facial expressions (Ekman \& Friesen, 2003), we decided to take surprise into consideration as a seventh facial expression for the rating.

2. One middle-aged woman did not return after completion of the first test session.

3. Young happy faces were not rated as younger than young neutral faces by young and older raters and were rated as older than young neutral faces by middle-aged raters; middle-aged happy faces were not rated as younger than middle-aged neutral faces by young raters; older happy faces were not rated as younger than older angry or older neutral faces by young raters; middle-aged fearful faces were not rated as older than middle-aged sad faces by young and middle-aged raters; older fearful faces were not rated as older than older sad faces by young, middleaged, and older raters, were not rated as older than older disgusted faces by middle-aged and older raters, and were not rated as older than older neutral faces by middle-aged raters (see Figure 5).

APPENDIXA

Percentages (Means and Standard Deviations) of Correct Identification of Facial Expressions in the Validation Sample

\begin{tabular}{|c|c|c|c|c|c|c|c|c|c|c|c|c|}
\hline & \multicolumn{12}{|c|}{ Facial Expression } \\
\hline & \multicolumn{2}{|c|}{ Happy } & \multicolumn{2}{|c|}{ Angry } & \multicolumn{2}{|c|}{ Fearful } & \multicolumn{2}{|c|}{ Sad } & \multicolumn{2}{|c|}{$\underline{\text { Disgusted }}$} & \multicolumn{2}{|c|}{ Neutral } \\
\hline & $M$ & $S D$ & M & $S D$ & $M$ & $S D$ & $M$ & $S D$ & $M$ & $S D$ & $M$ & $S D$ \\
\hline \multicolumn{13}{|l|}{ Young Female Raters } \\
\hline Young female faces & 99 & 3 & 94 & 5 & 85 & 14 & 85 & 16 & 81 & 14 & 91 & 11 \\
\hline Young male faces & 99 & 2 & 90 & 9 & 85 & 16 & 80 & 13 & 83 & 13 & 96 & 6 \\
\hline Middle-aged female faces & 99 & 3 & 90 & 8 & 86 & 16 & 85 & 12 & 83 & 15 & 89 & 13 \\
\hline Middle-aged male faces & 97 & 8 & 89 & 11 & 86 & 12 & 77 & 13 & 78 & 16 & 90 & 10 \\
\hline Older female faces & 96 & 7 & 84 & 12 & 83 & 16 & 83 & 10 & 74 & 14 & 87 & 11 \\
\hline Older male faces & 95 & 15 & 80 & 14 & 84 & 14 & 72 & 12 & 67 & 20 & 83 & 14 \\
\hline \multicolumn{13}{|l|}{ Young Male Raters } \\
\hline Young female faces & 97 & 11 & 89 & 16 & 85 & 16 & 86 & 17 & 76 & 19 & 93 & 13 \\
\hline Young male faces & 96 & 15 & 88 & 16 & 86 & 18 & 78 & 18 & 78 & 18 & 94 & 15 \\
\hline Middle-aged female faces & 96 & 10 & 84 & 16 & 86 & 17 & 82 & 18 & 75 & 19 & 86 & 17 \\
\hline Middle-aged male faces & 96 & 10 & 84 & 15 & 82 & 18 & 70 & 18 & 70 & 21 & 87 & 16 \\
\hline Older female faces & 96 & 12 & 78 & 19 & 83 & 16 & 74 & 18 & 65 & 17 & 82 & 19 \\
\hline Older male faces & 96 & 10 & 75 & 17 & 81 & 18 & 68 & 19 & 59 & 18 & 79 & 21 \\
\hline \multicolumn{13}{|l|}{ Middle-Aged Female Raters } \\
\hline Young female faces & 98 & 4 & 93 & 8 & 79 & 14 & 79 & 18 & 73 & 18 & 96 & 5 \\
\hline Young male faces & 98 & 6 & 90 & 9 & 81 & 14 & 72 & 21 & 75 & 19 & 95 & 10 \\
\hline Middle-aged female faces & 98 & 5 & 89 & 9 & 85 & 12 & 71 & 19 & 71 & 18 & 91 & 9 \\
\hline Middle-aged male faces & 98 & 4 & 92 & 8 & 81 & 15 & 69 & 19 & 64 & 21 & 92 & 9 \\
\hline Older female faces & 97 & 7 & 75 & 12 & 83 & 12 & 67 & 16 & 62 & 20 & 83 & 15 \\
\hline Older male faces & 98 & 4 & 77 & 9 & 78 & 13 & 62 & 18 & 53 & 17 & 81 & 17 \\
\hline \multicolumn{13}{|l|}{ Middle-Aged Male Raters } \\
\hline Young female faces & 98 & 6 & 86 & 12 & 81 & 18 & 82 & 14 & 74 & 17 & 87 & 13 \\
\hline Young male faces & 99 & 3 & 87 & 12 & 78 & 23 & 79 & 18 & 72 & 18 & 93 & 13 \\
\hline Middle-aged female faces & 99 & 2 & 86 & 12 & 83 & 17 & 75 & 15 & 72 & 16 & 84 & 16 \\
\hline Middle-aged male faces & 99 & 2 & 90 & 10 & 77 & 19 & 64 & 21 & 64 & 20 & 86 & 16 \\
\hline Older female faces & 98 & 3 & 73 & 15 & 78 & 19 & 70 & 15 & 68 & 14 & 79 & 17 \\
\hline Older male faces & 97 & 6 & 81 & 15 & 75 & 19 & 56 & 22 & 57 & 19 & 82 & 17 \\
\hline \multicolumn{13}{|l|}{ Older Female Raters } \\
\hline Young female faces & 99 & 2 & 81 & 15 & 82 & 13 & 82 & 17 & 70 & 22 & 88 & 11 \\
\hline Young male faces & 97 & 5 & 77 & 19 & 78 & 17 & 80 & 17 & 68 & 22 & 91 & 9 \\
\hline Middle-aged female faces & 98 & 5 & 76 & 14 & 83 & 16 & 74 & 18 & 67 & 16 & 81 & 15 \\
\hline Middle-aged male faces & 99 & 3 & 82 & 11 & 78 & 13 & 71 & 17 & 61 & 22 & 84 & 12 \\
\hline Older female faces & 95 & 11 & 67 & 18 & 83 & 12 & 75 & 15 & 64 & 18 & 78 & 19 \\
\hline Older male faces & 97 & 7 & 73 & 17 & 82 & 15 & 62 & 17 & 52 & 22 & 78 & 18 \\
\hline \multicolumn{13}{|l|}{ Older Male Raters } \\
\hline Young female faces & 93 & 14 & 76 & 16 & 76 & 19 & 72 & 18 & 68 & 20 & 88 & 15 \\
\hline Young male faces & 92 & 16 & 76 & 19 & 70 & 25 & 71 & 14 & 69 & 23 & 93 & 13 \\
\hline Middle-aged female faces & 91 & 16 & 70 & 20 & 74 & 22 & 66 & 20 & 64 & 21 & 85 & 15 \\
\hline Middle-aged male faces & 91 & 18 & 79 & 13 & 72 & 21 & 61 & 17 & 55 & 22 & 87 & 14 \\
\hline Older female faces & 89 & 18 & 58 & 21 & 69 & 23 & 62 & 22 & 56 & 20 & 80 & 20 \\
\hline Older male faces & 92 & 16 & 64 & 21 & 68 & 25 & 53 & 19 & 50 & 23 & 80 & 19 \\
\hline
\end{tabular}


APPENDIX B

Means and Standard Deviations of Perceived Age of Faces in the Validation Sample

\begin{tabular}{|c|c|c|c|c|c|c|c|c|c|c|c|c|}
\hline & \multicolumn{12}{|c|}{ Facial Expression } \\
\hline & \multicolumn{2}{|c|}{ Happy } & \multicolumn{2}{|c|}{ Angry } & \multicolumn{2}{|c|}{ Fearful } & \multicolumn{2}{|c|}{ Sad } & \multicolumn{2}{|c|}{ Disgusted } & \multicolumn{2}{|c|}{ Neutral } \\
\hline & $M$ & $S D$ & $M$ & $S D$ & $M$ & $S D$ & $M$ & $S D$ & $M$ & $S D$ & $M$ & $S D$ \\
\hline Young female faces & 25.4 & 2.35 & 25.4 & 2.89 & 26.8 & 2.03 & 25.9 & 2.08 & 25.2 & 2.51 & 25.1 & 2.42 \\
\hline Young male faces & 26.2 & 1.92 & 28.4 & 1.94 & 27.5 & 2.51 & 27.6 & 2.13 & 27.2 & 1.92 & 26.4 & 2.00 \\
\hline Middle-aged female faces & 46.7 & 2.95 & 47.3 & 3.24 & 48.1 & 2.88 & 48.5 & 2.95 & 46.8 & 3.63 & 47.4 & 3.10 \\
\hline Older female faces & 66.4 & 3.55 & 67.1 & 3.70 & 67.1 & 3.63 & 67.5 & 3.48 & 66.9 & 4.24 & 67.2 & 3.14 \\
\hline Older male faces & 69.6 & 3.78 & 69.7 & 3.78 & 70.5 & 3.44 & 70.9 & 3.77 & 69.8 & 3.64 & 69.6 & 4.06 \\
\hline \multicolumn{13}{|l|}{ Young Male Raters } \\
\hline Young female faces & 26.4 & 1.91 & 26.7 & 1.85 & 28.2 & 1.94 & 27.0 & 1.84 & 26.7 & 2.11 & 26.2 & 1.94 \\
\hline Young male faces & 26.6 & 2.05 & 28.2 & 2.19 & 28.1 & 2.24 & 27.9 & 2.13 & 27.7 & 2.29 & 26.7 & 1.97 \\
\hline Middle-aged female faces & 46.5 & 3.36 & 47.3 & 3.19 & 48.4 & 2.87 & 48.2 & 3.31 & 47.1 & 3.39 & 47.1 & 3.08 \\
\hline Young female faces & 26.5 & 2.89 & 27.1 & 2.81 & 29.2 & 2.75 & 26.7 & 2.84 & 27.2 & 2.86 & 26.0 & 3.33 \\
\hline Young male faces & 27.5 & 2.93 & 30.1 & 2.80 & 29.6 & 3.01 & 29.2 & 2.91 & 29.3 & 3.15 & 27.0 & 3.07 \\
\hline Middle-aged female faces & 47.6 & 6.55 & 47.9 & 3.23 & 49.0 & 2.92 & 50.3 & 5.15 & 48.3 & 3.02 & 49.0 & 5.33 \\
\hline Middle-aged male faces & 49.1 & 3.38 & 50.2 & 3.32 & 51.5 & 3.68 & 50.6 & 3.16 & 51.2 & 2.75 & 48.8 & 3.15 \\
\hline Older female faces & 67.3 & 3.97 & 67.7 & 3.62 & 68.4 & 3.95 & 68.9 & 4.31 & 67.9 & 3.82 & 68.3 & 3.35 \\
\hline Older male faces & 68.3 & 4.21 & 68.8 & 3.65 & 69.4 & 3.73 & 69.4 & 3.53 & 69.5 & 3.92 & 69.1 & 3.30 \\
\hline \multicolumn{13}{|l|}{ Middle-Aged Male Raters } \\
\hline Young female faces & 27.1 & 3.96 & 28.2 & 3.88 & 30.1 & 4.28 & 28.4 & 4.24 & 28.2 & 4.38 & 26.3 & 4.54 \\
\hline Young male faces & 27.7 & 3.52 & 30.7 & 4.29 & 29.6 & 3.40 & 29.6 & 3.36 & 29.9 & 3.61 & 27.5 & 3.40 \\
\hline Middle-aged female faces & 46.4 & 4.02 & 48.4 & 4.71 & 48.7 & 4.18 & 49.1 & 4.54 & 47.2 & 4.03 & 48.0 & 3.89 \\
\hline Middle-aged male faces & 48.1 & 4.00 & 49.4 & 4.58 & 50.6 & 4.13 & 49.5 & 4.66 & 50.1 & 4.19 & 47.9 & 3.77 \\
\hline Older female faces & 68.6 & 4.71 & 70.0 & 5.04 & 71.4 & 4.78 & 71.8 & 4.90 & 71.0 & 4.71 & 70.9 & 4.51 \\
\hline Older male faces & 69.5 & 4.55 & 71.2 & 4.30 & 71.7 & 3.96 & 71.7 & 4.21 & 71.7 & 4.27 & 70.6 & 4.36 \\
\hline \multicolumn{13}{|l|}{ Older Male Raters } \\
\hline Young female faces & 28.2 & 4.49 & 30.2 & 5.10 & 32.6 & 5.14 & 30.3 & 4.59 & 30.9 & 4.48 & 27.9 & 4.77 \\
\hline Young male faces & 29.6 & 4.34 & 34.0 & 4.40 & 32.6 & 4.18 & 32.8 & 4.31 & 33.2 & 4.44 & 29.8 & 4.58 \\
\hline Middle-aged female faces & 45.5 & 3.86 & 48.9 & 4.52 & 50.0 & 3.85 & 50.4 & 4.18 & 49.1 & 4.38 & 48.6 & 3.69 \\
\hline Middle-aged male faces & 48.8 & 2.56 & 51.4 & 3.57 & 53.4 & 3.78 & 52.1 & 3.19 & 52.8 & 3.18 & 49.4 & 3.46 \\
\hline Older female faces & 67.4 & 5.09 & 68.2 & 5.10 & 69.7 & 5.62 & 70.1 & 5.18 & 69.5 & 5.11 & 68.5 & 4.64 \\
\hline Older male faces & 69.0 & 4.14 & 69.8 & 4.30 & 70.3 & 5.14 & 71.1 & 4.24 & 70.3 & 4.70 & 67.0 & 3.80 \\
\hline
\end{tabular}

(Manuscript received March 27, 2009;

revision accepted for publication June 6, 2009.) 